\title{
Ultrasonic Measurement of Cylinder Expansion at Pressures to 40 Kilobars
}

\author{
Peter L. M. Heydemann and James C. Houck \\ Institute for Basic Standards, National Bureau of Standards, Washington, D.C. 20234
}

(September 12, 1966)

\begin{abstract}
For the accurate determination of internal pressures in solid-filled piston and cylinder devices the effective area and its change with pressure are very significant factors. The computation of the change of area with pressure from elastic theory leads however to considerable uncertainties.

The present paper describes how ultrasonic measurements made in solid-filled piston and cylinder devices to 45 kilobars are combined with measurements of the lengths of the samples as function of pressure and with ultrasonic measurements under hydrostatic pressure to yield values for cylinder expansion. The results are compared with values obtained from modified elastic theory.
\end{abstract}

Key Words: Compressibility, cylinder distortion, high pressure, nickel, tellurium, ultrasonics.

\section{Introduction}

Several solid filled piston and cylinder devices with rotatable pistons have been used in this laboratory to determine transition pressures at pressures up to 45 kbar. The internal pressure was computed as the ratio of ram force on the piston to effective area of the cylinder. Appropriate corrections were made for friction between moving and stationary parts and for distortion of the cylinder. An estimate of the change of the effective area of the cylinder with internal pressure was calculated using equations developed from elastic theory for long thick-walled cylinders subjected to internal pressure. These relations were modified using the solution to the tangential strain problem for an infinite cylinder with a semi-infinite zone of internal pressure [1]. ${ }^{1}$

The estimated uncertainty in this calculation of the area is two parts per thousand at $25 \mathrm{kbar}$ and four parts per thousand at $40 \mathrm{kbar}$ provided that the elastic theory on which the calculation is based accurately describes the problem. This uncertainty is sufficiently large to warrant some effort in a direct determination of the change of area of the cylinder with internal pressure. This paper describes such a direct determination by means of ultrasonic measurements on a sample contained in the cylinder.

The ultrasonic measurements are used to determine the compressibility of the sample as a function of pressure. From this the length, which the sample would have in a rigid cylinder at any given pressure, is computed. This hypothetical length will be compared with a sample length obtained from a direct

'Figures in brackets indicate the literature references at the end of this paper. measurement at the same pressure. The ratio of these two lengths is a measure of the expansion of the cylinder.

\section{Definition of Expansion Factor}

We assume a mean value for the expansion of the cylinder over the pressurized length (fig. 1) and we define as the expansion factor:

$$
\alpha(p)=\frac{\text { mean expanded area of the cylinder } A_{\mathrm{ex}}}{\text { nonexpanded area of the cylinder } A_{0}} .
$$

$\alpha(p)$ is a function of pressure. The relative change of the area of the cylinder with pressure is

$$
\frac{\Delta A}{A_{0}}=\frac{A_{\mathrm{ex}}-A_{0}}{A_{0}}=\alpha(p)-1 .
$$

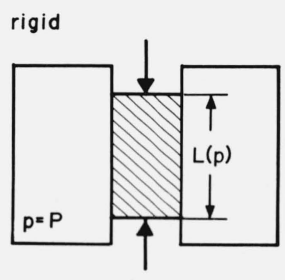

non-rigid
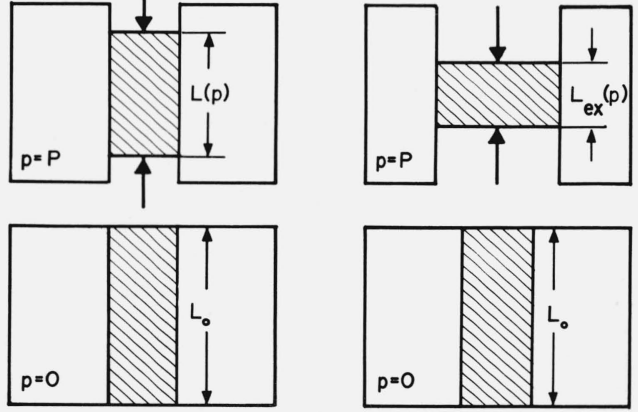

FIGURE 1. Deformation of sample under pressure with and without deformation of cylinder. 
We further assume that the pressure is uniform throughout the sample, that the end faces of the sam. ple are plane, and that the cylinder remains straight.

The expansion of the cylinder does not change the volume $V(p)$ which the sample assumes at pressure $p$, so that

$$
V(p)=A_{0} L_{0}(1-K p)=A_{\text {ex }} L_{\text {ex }}(p)
$$

where $L_{\mathrm{ex}}(p)$ is the length of the sample in the expanded cylinder at pressure $p, L_{0}$ is the sample length at zero pressure, and $K$ is the compressibility of the sample (see fig. 1). Whence follows

$$
\alpha(p)=\frac{A_{\mathrm{ex}}}{A_{0}}=\frac{L_{0}(1-K(p) \cdot p)}{L_{\mathrm{ex}}(p)}=\frac{L(p)}{L_{\mathrm{ex}}(p)}
$$

According to eq (3) the distortion of the cylinder and the change of area could then be obtained from a measurement of the actual length of the sample and a computation of the uniaxial compression of the sample in a rigid-walled cylinder based on the compressibility $K(p)$ of the sample material.

\section{Determination of Sample Length $L(p)$ for Uniaxial Compression}

At low pressures, where the compressibility of the sample placed in a rigid cylinder is constant, its length $L(p)$ is

$$
L(p)=L_{0}(1-K p) .
$$

Generally $K$ is a function of pressure and, if this function is known, $L(p)$ may be computed from

$$
L(p)=L_{0}\left(1-\int_{0}^{p} K(p) d p\right) .
$$

Although data of compressibility versus pressure have been reported for a number of materials, the agreement between different sources for the same material is often very disappointing. It is desirable to use materials with small compressibilities to reduce the uncertainty in the determination of $L(p)$.

The choice of materials is also narrowed down by the fact that the present purpose requires a material with low yield strength to ensure a more nearly hydrostatic stress pattern.

Typical uncertainties in $L(p)$ caused by an uncertainty of 10 percent in the compressibility of the material are 0.1 percent for nickel at $20 \mathrm{kbar}$ and 3 percent for the more compressible tellurium at 40 kbar.

In 1957 R. K. Cook published a paper [2] dealing with the variation of the elastic constants and static strains with hydrostatic pressure and their calculation from ultrasonic measurements. The final equation reported in that paper relates the sample length at the hydrostatic pressure $p$ with the fundamental resonance frequencies of the same sample measured as a function of pressure for longitudinal and shear waves propagating along the axis of the cylindrical sample:

$$
\frac{L_{0}}{L(p)}=1+\frac{1+\Delta}{3 h_{0}} \int_{0}^{p} \frac{d p}{f_{1}^{2}-\frac{4}{3} f_{s}^{2}}
$$

where $L_{0}$ is the length of sample at zero pressure

$L(p)$ is the length of sample at pressure $p$ $h_{0}=4 L_{0}^{2} \rho_{0}$

$f_{1}, f_{s}$ are the resonance frequencies for longitudinal and shear waves, and

$$
\Delta=\frac{K_{\mathrm{is}}}{K_{\mathrm{ad}}}-1
$$

where $K_{\text {ad }}$ is the adiabatic bulk compressibility

$K_{\text {is }}$ is the isothermal bulk compressibility

$h_{0}\left(f_{1}^{2}-\frac{4}{3} f_{s}^{2}\right)$ is essentially the adiabatic bulk modulus $B_{\text {ad }}=K_{\text {ad }}^{-1}$.

In the derivation of eq (6) two approximations were made

$$
\frac{\left(1-\frac{1}{3} p K(p)\right)^{2}}{(1-p K(p))} \approx 1
$$

and

$$
\frac{1}{1-\frac{1+\Delta}{3} \int_{0}^{p} K(p) d p} \approx 1+\frac{1+\Delta}{3} \int_{0}^{p} K(p) d p
$$

In the case of very compressible materials like tellurium the first approximation leads to a value of $L(p)$ that is too large by a few tenths of 1 percent at $40 \mathrm{kbar}$. An error of similar magnitude but opposite sign is introduced if $\Delta$ in eq (6) is neglected. The second approximation can be avoided by rewriting eq (6). With less compressible materials like, for example, nickel, both corrections amount to less than 0.1 percent in $L(p)$. This indicates again that materials with low compressibility are to be preferred for such measurements.

Equation (6) permits the computation of the length of a sample subjected to hydrostatic pressure from the resonance frequencies obtained over the entire pressure range. The same equation less the factor 3 in the denominator in front of the integral holds for a sample contained in a rigid cylinder and compressed longitudinally. 
If the measurement is made in a nonrigid cylinder where the length of the sample is reduced by $\alpha^{-1}$ due to cylinder expansion, eq (6) has to be corrected by multiplying $h_{0}=4 L_{0}^{2} \rho_{0}$ with $\alpha(p)^{-2}$. Since $\alpha(p)$ is a function of pressure it has to be written under the integral. Equation (6) for a nonrigid cylinder then becomes

$$
\frac{L_{0}}{L(p)}=1+\frac{1+\Delta}{h_{0}} \int_{0}^{p} \frac{\alpha^{2}(p) d p}{f_{1}^{2}-\frac{4}{3} f_{s}^{2}} .
$$

$\alpha^{2}(p)$ does not differ appreciably from unity. To facilitate the solution of eq (10) we assume that $\alpha(p)$ is a linear function of $p$

$$
\alpha(p)=1+\beta p ; \beta=\frac{\delta \alpha}{\delta p},
$$

and we use an estimated value for $\beta$ in the actual numerical evaluation of this and related equations.

$L(p)$ obtained from eq (10) can be combined with an $L_{\text {ex }}(p)$ obtained from dial gage measurements of the sample length as described in section 6 to yield the expansion factor:

$$
\alpha_{1}(p)=\frac{L_{0}}{L_{\mathrm{ex}}(p)} \frac{1}{1+\frac{1+\Delta}{h} \int_{0}^{p} \frac{\alpha^{2} d p}{f_{1}^{2}-\frac{4}{3} f_{s}^{2}}} .
$$

Instead of using ultrasonic measurements in a solidfilled piston cylinder device to determine $L(p)$, a sample length $L^{B}(p)$ could be determined under hydrostatic conditions with higher precision and then be converted to the hypothetical length $L(p)$ with the help of the relation

$$
\frac{L_{p}^{B}-L_{0}^{B}}{L_{0}^{B}}=\frac{L(p)-L_{0}}{3 L_{0}},
$$

and the equation for $\alpha(p)$ is then

$$
\alpha_{2}(p)=\frac{L_{0}}{L_{\mathrm{ex}}(p)}\left[\frac{3}{1+\frac{1+\Delta}{3 h} \int_{0}^{p} \frac{d p}{f_{1}^{B^{2}}-\frac{4}{3} f_{s}^{B^{2}}}}-2\right]
$$

The superscript $B$ in these equations denotes values obtained under hydrostatic conditions.

Equations (12) and (13) are not very sensitive to errors made in the determination of the frequencies or the compressibility derived from them as long as materials with low compressibility are used. The main source of error is the determination of $L_{\mathrm{ex}}(p)$. In a particular experiment to be described below an error of $25 \mu \mathrm{m}$ in the determination of $L_{\mathrm{ex}}(p)$ would cause an error in the change of area, $\Delta A$, of more than 30 percent.

\section{Determination of Actual Sample Length $L_{\mathrm{ex}}(p)$ from Ultrasonic Measurements}

In equations (11) and (13) the sample lengths computed for the rigid-walled cylinder were combined with an actual sample length obtained from dial gage readings. If, from ultrasonic measurements under hydrostatic conditions, both the shear and the longitudinal wave velocity $(v)$ are known, these, together with the resonance frequencies or the times of flight $(\tau)$ through the sample in the solid-filled cylinder, can be used to determine the actual length of the sample:

$$
L_{\mathrm{ex}}(p)=\frac{v}{2 f}=v \tau \text {. }
$$

Two values for $L_{\mathrm{ex}}(p)$ are obtained; one from shear waves and the other from longitudinal waves. The precision required in $f$ is rather high. An uncertainty of less than 0.1 percent is necessary and 0.01 percent is desirable at the highest pressures.

The two values thus obtained for $L_{\mathrm{ex}}(p)$ can be combined with the two values for $L(p)$ used in eqs (11) and (13) in four different ways to yield:

$$
\begin{gathered}
\alpha_{3}(p)=\frac{L_{0} f_{1}}{L_{0}^{B} f_{1}^{B}} \frac{1+\frac{1+\Delta}{3 h_{0}} \int_{0}^{p} \frac{d p}{f_{1}^{B^{2}}-\frac{4}{3} f_{s}^{B^{2}}}}{1+\frac{1+\Delta}{h_{0}} \int_{0}^{p} \frac{\alpha_{2} d p}{f_{1}^{2}-\frac{4}{3} f_{s}^{2}}} \\
\alpha_{4}(p)=\frac{L_{0} f_{s}}{L_{0}^{B} f_{s}^{B}} \frac{1+\frac{1+\Delta}{3 h_{0}} \int_{0}^{p} \frac{d p}{1+\frac{1+\Delta}{h_{0}} \int_{0}^{p} \frac{\alpha_{2}{ }^{2}-\frac{4}{3} f_{s}^{B^{2}}}{f_{1}^{2}-\frac{4}{3} f_{s}^{2}}}}{\alpha_{5}(p)=\frac{L_{0} f_{1}}{L_{0}^{B} f_{1}^{B}}\left[1-\frac{2}{3} \frac{1+\Delta}{h_{0}} \int_{0}^{p} \frac{d p}{f_{1}^{B^{2}}-\frac{4}{3} f_{s}^{B^{2}}}\right]} \\
\alpha_{6}(p)=\frac{L_{0} f_{s}}{L_{0}^{B} f_{s}^{B}}\left[1-\frac{2}{3} \frac{1+\Delta}{h_{0}} \int_{0}^{p} \frac{d p}{f_{1}^{B^{2}}-\frac{4}{3} f_{s}^{B^{2}}}\right]
\end{gathered}
$$

The expressions in brackets are very close to unity and, especially with materials of low compressibility, do not contribute very much to the total uncertainty. $L_{0}$ and $L_{0}^{B}$, the initial sample lengths, can be measured with sufficient precision. The main source of error is in measuring the resonance frequencies. While the frequencies in hydrostatic environments can often be reproduced to better than 0.01 percent at $20 \mathrm{kbar}$ no such claim can be made for our solid-filled cylinder assemblies where a scatter of the data of up to 1 percent was encountered. In planned future work a 
very definite improvement is expected from improved ultrasonic techniques and choice of more suitable materials.

\section{Description of Pressure Equipment and Pressure Determination}

A diagrammatic sketch of the equipment is shown in figure 2. The cylinders used were made from cemented tungsten carbide with 6 percent cobalt. Radial support was provided by hardened steel support rings with an interference fit. Axial support was provided by end-loading applied to the carbide cylinder through a bridge plate resting on extensions of a thick cylinder wall of a ram with a $15 \mathrm{~cm}$ diam piston. This ram operated on the high pressure piston through a hole in the bridge plate. Rotation of the high pressure piston and $15 \mathrm{~cm}$ diam piston is accomplished by a handle passing between the cylinder wall extensions. Both the end loading force and the $15 \mathrm{~cm}$ diam piston force are supplied by the large press in

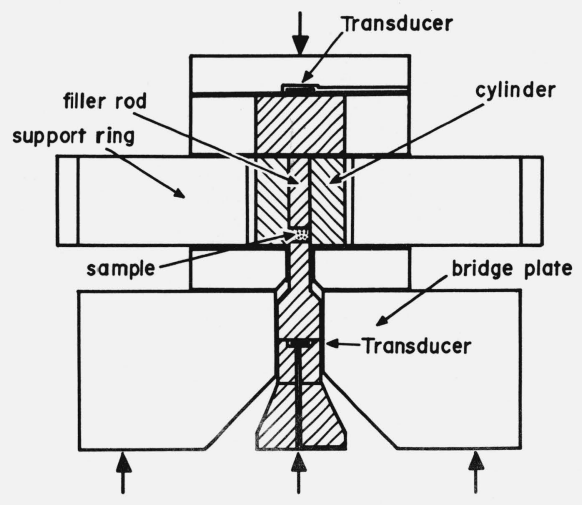

FIGURE 2. Long cylinder.

Tungsten carbide parts hatched. Sample marked by dotted area.

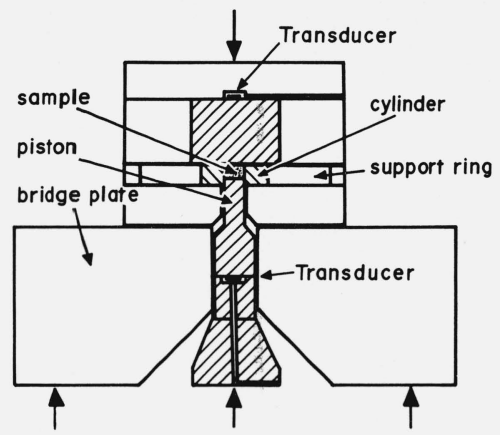

FiguRE 3. Short cylinder.

Tungsten carbide parts hatched. Sample marked by dotted area. which this equipment is inserted. Transition pressure measurements were made in this equipment using a high pressure cylinder $5 \mathrm{~cm}$ long with a bore of $1.26 \mathrm{~cm}$. Quartz transducers were mounted as shown in figure 2 . In the $5 \mathrm{~cm}$ long cylinder a carbide filler rod was used to give the desired sample length for an entrance of the movable piston of about $0.1 \mathrm{~cm}$ at zero ram force. This additional member in the path of the ultrasonic signal was undesirable. It was later eliminated by the use of a $1 \mathrm{~cm}$ long cylinder as shown in figure 3.

Piston displacement was measured with a dial gage indicator mounted on the cylinder rim and bearing against an extension of the rotation handle. The displacement read is the sum of the movement of the sample end of the high pressure piston, the compression of the high pressure piston, and the compression of the carbide pieces which back up the piston. The movement of the sample end of the high pressure piston is due to compression of the sample, expansion of the cylinder, and, if used, the carbide filler rod.

In the $5 \mathrm{~cm}$ long cylinder a sample was compacted from tellurium powder with a particle size of $50 \mu \mathrm{m}$ or less. The length after compaction to $40 \mathrm{kbar}$ was $0.6858 \mathrm{~cm}$. Conical steel rings were used to prevent extrusion. The transition pressure with rotation (approximately two degree oscillation) was determined to be $40 \mathrm{kbar}$. In the $1 \mathrm{~cm}$ long cylinder a nickel sample, machined to fit, was used for measurements to $20 \mathrm{kbar}$. For the ultrasonic measurements the equipment was operated without rotation.

The determination of internal pressure requires correction for friction and the determination of sample length requires correction of sample length for compression of various members. Figure 4 shows a typical curve of uncorrected dial gage reading against uncorrected increasing and decreasing pressure. The dashed line to $\mathrm{A}$ is the calculated change in dial gage reading with pressure due to the compressibility

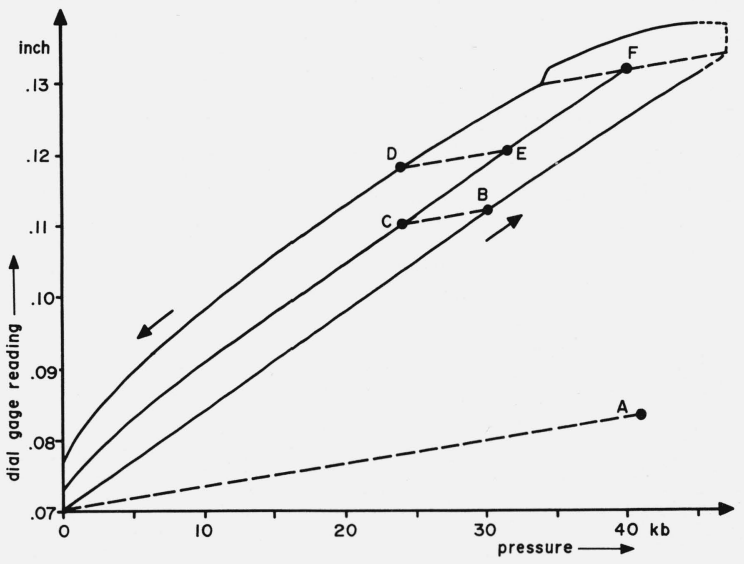

FIGURE 4. Change of length of tellurium sample as indicated by dial gage reading and correction to internal pressure. 
of the carbide high pressure piston and its supporting pieces. A modified mean curve for the pressure on the tellurium is drawn to point $\mathrm{F}$, the start of a transition. The corrected pressure is obtained by following a line with a slope equal to that of line "A" from an observed point B or D to the intersection with the modified mean curve (points $\mathrm{C}$ or E) respectively. These corrected pressures are used with the observed frequencies for the ultrasonic computations.

\section{Determination of the Measured Sample Length, $L_{\mathrm{ex}}(p)$}

The measured sample length, $L_{\mathrm{ex}}$, is obtained by applying a correction to the dial gage reading for the compression of the various carbide members. For pressures to $20 \mathrm{kbar}$ a constant value of Young's modulus for the carbide of $5.93 \times 10^{3} \mathrm{kbar}$ was used. For pressures from 20 to 40 kbar a decreasing Young's modulus was used.

The effects of uncertainties in $L_{\mathrm{ex}}$ on $\alpha$ are computed for a 5 percent uncertainty in Young's modulus and for a 10 percent uncertainty in the estimate of effective length of the compressible carbides including the tapered piston and the backup pieces of various crosssections. These effects on $\alpha(p)$ are larger in the tellurium measurements than in the nickel measurements primarily because of the greater length of compressible carbide in the tellurium measurement and secondarily because of the shorter sample length of the tellurium. These uncertainties in $\alpha(p)$ due to $L_{\mathrm{ex}}$ are shown in figures 7 and 8 and will be discussed later.

\section{Derivation of Expansion Factor $\alpha(p)$ from Elastic Theory}

The expansion of the bore of a long thick-walled cylinder due to internal pressure $p$ is

$$
\frac{\Delta d}{d}=\frac{p}{E} \frac{(1-\mu)+(1+\mu) W^{2}}{W^{2}-1}
$$

where $E$ is Young's modulus, $\mu$ is Poisson's ratio, and $W$ is the ratio of the outer diameter to inner diameter of the cylinder.

The cylinder used with the tellurium had a wall ratio, $W$, of 4 and a length of $5 \mathrm{~cm}$ with only $0.686 \mathrm{~cm}$ or about one radius of that length exposed to internal pressure.

A proportionality factor, $F$, to be applied to eq (19) for this pressure distribution is computed using values of tangential strain at the inner surface of a cylinder for a semi-infinite zone of internal pressure from the "Thick Walled Cylinder Handbook" [1]. For a long cylinder with internal pressure over a length equal to one radius starting at one end, the proportional expansion is computed by assuming a mirror image of the cylinder at the pressurized end of the cylinder. In Figure 5 curves $(a$ and $b$ ) are drawn for semi-infinite

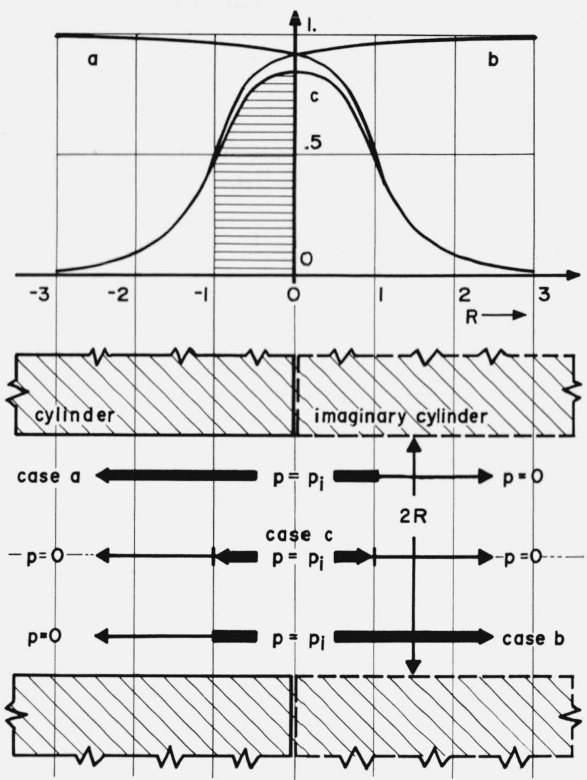

FiguRE 5. Derivation of cylinder expansion from the distortion caused by reflected semi-infinite zones of internal pressure.

pressure zones starting at the original and reflected pressure discontinuities. Curve $c$ is the product of " $a$ " and " $b$ " and is an approximation for the factor for a pressurized zone of length $2 R$ in a long cylinder.

A mean value of 0.75 is taken to apply to the expansion calculated from eq (19). $\quad \alpha(p)$ is computed using the above factor and a Young's modulus of $5.79 \times 10^{3}$ kbar for pressures 0 to $20 \mathrm{kbar}$ and a decreasing Young's modulus for pressures of 20 and $40 \mathrm{kbar}$. The computed $\alpha(p)$ for the cylinder used with the tellurium sample is shown in figure 7 . The effects of a 5 percent uncertainty in $E$ and a 33 percent uncertainty in $F$ are shown.

The $1 \mathrm{~cm}$ long carbide cylinder used with the nickel sample has a wall ratio $W=3$. Equation (19) is used neglecting the increase in effective $W$ due to the steel rings and the variation of the expansion of this short section from that of a long cylinder. For a value for $E$ of $5.97 \times 10^{3} \mathrm{kbar}$ and a $\mu$ of 0.25 :

$$
\begin{aligned}
& \frac{\Delta d}{d}=0.00503 \text { at } 20 \mathrm{kbar} \\
& \frac{\Delta A}{A}=\alpha-1=0.0101 \text { at } 20 \mathrm{kbar} .
\end{aligned}
$$

An estimated uncertainty of 5 percent in $E$ results in a 5 percent uncertainty in $\alpha-1$ and this is shown in the value of $\alpha$ in figure 8 . No value is included for the uncertainty introduced by applying the theory for elastic distortion of long cylinders to a short section and neglecting the friction effects at the end faces. 


\section{Experimental Determination of Cylinder Expansion Factors}

For the ultrasonic measurements in the solid-filled piston and cylinder assemblies the signal had to be generated outside the pressurized zone, radiated through the sample and picked up again outside the pressurized zone. Since plane and parallel interfaces along the ultrasonic path are necessary for all precise measurements, the signal could only be radiated along the longitudinal axis of the apparatus. Figures 2 and 3 show how the $10 \mathrm{MHz}$ quartz transducers were arranged. One transducer was used for shear, the other for longitudinal waves. Part of the signal originating from a transducer is reflected at the tungsten carbide-sample interface, the rest travels through the sample and undergoes another reflection at the sampletungsten carbide interface. The returning echoes plus usually several artifacts were picked up by the same transducer. The signal was then amplified and displayed on the screen of an oscilloscope without demodulation.

The delay time caused by the sample was measured in two ways: At high attenuations a short pulse was used and the time between the reflection from the front (tungsten carbide-sample) interface and the rear (sample-tungsten carbide) interface was measured by displaying the rf-pulse on the screen of an oscilloscope and using the variable sweep delay to let the pulses successively coincide with a mark on the screen. The transit times could thus be determined with a sensitivity of $\pm 20 \mathrm{~ns}$. At low values of attenuation in the samples the phase comparison method developed by McSkimin [3] was used. The pulse length was adjusted to provide sufficient overlap of succeeding pulses and the rf-frequency was adjusted for destructive interference. At constant pressure the critical frequencies could usually be reset to $\pm 1 \mathrm{kHz}$ at 10 to $12 \mathrm{MHz}$.
The electronic equipment consisted of an rf-cwgenerator, a gated amplifier and, on the receiver side, a wide-band rf-amplifier and a wide band oscilloscope. The transducers were connected to the circuit through a tuned transformer bridge.

The same electronic equipment was also used for ultrasonic measurements with a nickel sample under hydrostatic pressure. In this case only the phase comparison method was used. The sample was cemented between two fused quartz buffer rods with a shear and a longitudinal mode transducer attached to either end.

Figure 6 shows a typical plot of transit time versus uncorrected pressure obtained in a solid-filled cylinder. The large amount of hysteresis is due to the friction between the piston and the cylinder. In section 5 we have already described how the uncorrected pressure is related to the internal pressure. This relation is now used to re-plot the ultrasonic data versus internal pressure. The result is, in the case of shear waves in tellurium, a single curve for increasing and decreasing pressure. In some cases the results were less satisfactory. Transit time data are read from the final curves at intervals of 1 kbar to be used for the computation of the cylinder expansion.

The results of two such computations are shown in figures 7 and 8 . Figure 7 is for the long cylinder shown in figure 2 filled with sintered tellurium powder compacted at $40 \mathrm{kbar}$. The expansion factor computed from elastic theory is shown as a solid line. The uncertainty is schematically indicated by the vertically hatched area. Data for the expansion factor computed from ultrasonic measurements and measurements of sample length are entered as black dots. The total of the estimated uncertainties of the measured length is schematically indicated by the horizontally hatched area. The estimated uncertainty introduced by the ultrasonically measured $L(p)$ is considerably smaller. Also entered in figure 7 are the adiabatic compres-

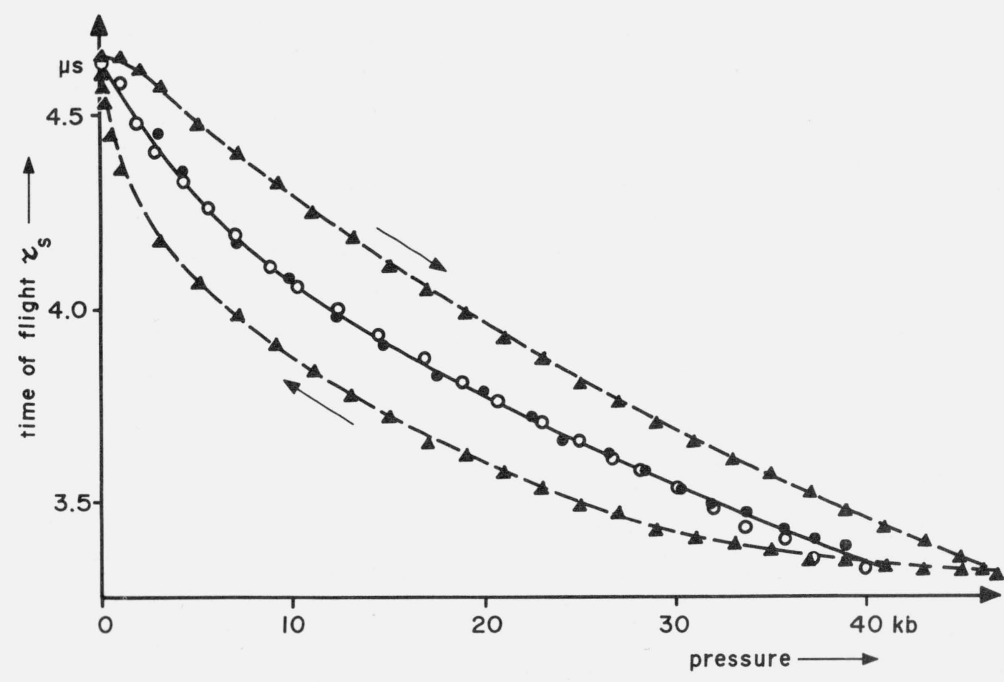

Figure 6. Time of flight for shear waves in tellurium as function of increasing, decreasing and corrected internal pressure. 


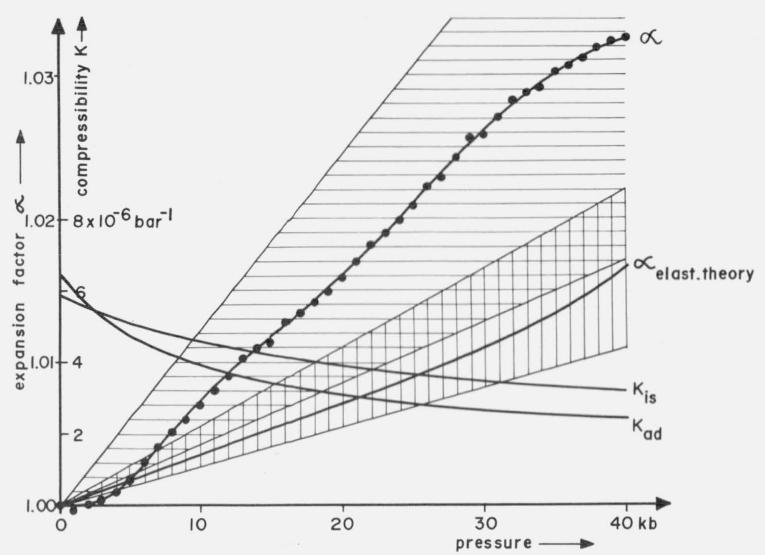

Figure 7. Cylinder expansion with tellurium sample in long cylinder.

Areas of uncertainty hatched.

sibility $K_{\text {ad }}$ obtained from the ultrasonic measurement and the isothermal compressibility $K_{\text {is }}$ obtained from the dial gage reading. The latter includes the effect of cylinder expansion and is therefore not a correct value.

The overall uncertainty in this determination is extremely large. The greater part of the uncertainty is due to the determination of $L_{\mathrm{ex}}(p)$ from the dial gage measurements. This fact emphasizes the need for an improved way to measure the actual length of the sample under pressure.

Figure 8 presents the results obtained with a short cylinder filled with nickel. The uncertainties are indicated in the same way as in figure 7 . The overall uncertainties are relatively much smaller. This is partly due to the improved design of the cylinder and partly to the smaller compressibility of the sample. The precision of the ultrasonic measurements was improved over that with tellurium. Consequently the disagreement between the measured and the computed values for $\alpha$ is much smaller. Again the compressibilities are entered.

\section{Conclusion}

The experimentally determined expansions for both the long and the short cylinder are larger than the

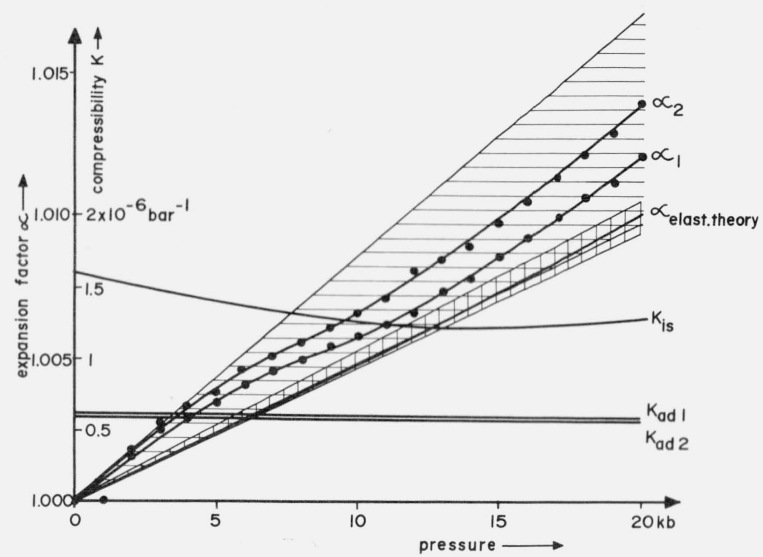

FIGURE 8. Cylinder expansion with nickel sample in short cylinder. Areas of uncertainty hatched.

expansions computed from elastic theory. The same relation was reported by Bridgman [4] for his experimental determinations of cylinder distortion under various conditions. Therefore, a more rigorous computation of elastic distortion of short cylinders, including end effects and other modifications, seems to be desirable. While this mathematical solution does not seem to be very near at hand, the main effort will have to be in the direction of improved ultrasonic measurements with carefully selected materials in various piston-cylinder devices. The application of ultrasonic measurements to the determination of cylinder distortion as proposed in this paper will then lead to more meaningful and more precise data.

\section{References}

[1] P.,P. Kadkowski, J. I. Bluhm, O. L. Bowie, Watertown Arsenal Laboratory, "Thick-Walled Cylinder Handbook," figures 6A and $6 \mathrm{~B}$.

[2] R. K. Cook, J. Acoust. Soc. Am. 29, 445 (1957).

[3] H. J. McSkimin, J. Appl. Phys. 24, 988 (1953).

[4] P. W. Bridgman, Proc. Am. Acad. Arts Sci. 74, 1 (1940).

(Paper 71C1-439) 